\section{Dez anos de epidemia do HIV- \\ AIDS em maiores de 60 anos no \\ Distrito Federal - Brasil}

\section{Ten years of HIV-AIDS epidemic in more than 60 years in Federal District - Brazil}

Maria Liz Cunha de Oliveira ${ }^{1,11}$

Leidijany Costa Paz'

Gislane Ferreira de Melo"

I Secretaria de Saúde do Distrito Federal.

" Universidade Católica de Brasília.

\section{Resumo}

Introdução: O aumento progressivo no número de casos de HIV/AIDS em idosos traz a necessidade de estudos sobre as especificidades deste fenômeno por região. Objetivo: Descrever as características dos casos de AIDS em indivíduos com idade $\geq 60$ anos ou mais no Distrito Federal - Brasil. Método: Realizou-se um estudo de série temporal, com dados secundários do SINAN/AIDS, no período de Janeiro de 1999 a dezembro 2009. Resultado: Neste período foram diagnosticados 4258 novos casos de AIDS; destes, 89 (2,0\%) são idosos. Embora seja uma porcentagem pequena, o crescimento anual foi contínuo. A categoria de exposição mais frequente foi a de heterossexual, a faixa etária mais acometida foi de 60 a 69 anos com $71(79,8 \%)$ casos, e a proporção de casos homem/mulher vem mudando ao longo dos anos, sendo que em 1999 era de 1:1 e, em 2006, de 0,7:1. Conclusões: A epidemia de HIV/AIDS pode ser considerada estável entre os idosos no Distrito Federal.

Palavras-chave: Síndrome da Imunodeficiência Adquirida. AIDS. Idoso. Epidemiologia. Doenças sexualmente transmissíveis. DST. 


\section{Abstract}

Introduction: The progressive increase in number of HIV/AIDS cases in old age brings the necessity of studies about the specificities of this phenomenon by region. Objective: To identify the epidemiology in AIDS cases diagnosed in Distrito Federal Brasil, in individuals aged $\geq 60$ years old. Methods: We conducted a time serie study with secondary data from SINAN/AIDS for the period January 1999 to December 2009. Results: In this period, 4,258 new cases of AIDS were of whom $89(2.0 \%)$ are elderly. Although a small percentage, the annual growth was continuous. The most frequent exposure category was heterosexual, the age group most affected was 60 and 69 years, with $71(79.8 \%)$ cases; the proportion of cases man/woman has changed over the years, in 1999 was 1:1 and in 2006, 0,7:1. Conclusion: The HIV/AIDS epidemic among the elderly can be seen stable on the Distrito Federal.

Keywords: Acquired Immunodeficiency Syndrome. AIDS. Aged. Epidemiology. Sexually transmitted diseases. STD.

\section{Introdução}

No Brasil, o ritmo de crescimento da população idosa tem sido sistemático e consistente. Em 2009, o país contava com uma população de cerca de 21 milhões de pessoas de 60 anos ou mais de idade. No período de 1999 a 2009, o número de idosos no conjunto da população passou de $9,1 \%$ para $11,3 \%{ }^{1}$.

Com uma taxa de fecundidade abaixo do nível de reposição populacional, combinada ainda com outros fatores, tais como os avanços da tecnologia, especialmente na área da saúde, atualmente o grupo de idosos ocupa um espaço significativo na sociedade brasileira. Estima-se que, nos próximos vinte anos, o número de idosos brasileiros poderá ultrapassar os 30 milhões, representando $13 \%$ da população ${ }^{1}$.

O envelhecimento populacional torna a saúde dos idosos um importante foco de atenção. Os "idosos", segundo a Organização Mundial da Saúde (OMS), são pessoas nas faixas etárias de 60 a 100 anos de idade e mais, que vivem em países em desenvolvimento ${ }^{2}$. Esta faixa etária abrange um período de mais de 40 anos, compreendendo, além de vivências muito diferenciadas, pessoas em pleno vigor físico e mental e outras em situações de dependência ${ }^{3}$.

Estudo sobre "Saúde Sexual e os Novos Idosos" ${ }^{4}$ chama a atenção para a mudança sociocultural das atitudes relativas à sexualidade e ao envelhecimento, e vem desafiando o estereótipo tradicional da "velhice assexuada", na medida em que a função sexual passou a ser vista como um componente vital para se alcançar um envelhecimento de sucesso em geral. Os fatores-chave que consolidam esta mudança incluem os recentes avanços da indústria farmacêutica, que permitem o prolongamento da vida sexual ativa, junto com mudanças de atitudes e comportamentos sexuais das pessoas mais velhas, em associação com a desmistificação do sexo, e tornam as pessoas idosas mais vulneráveis às doenças sexualmente transmissíveis (DST). Dentre elas, a infecção pelo vírus da 
imunodeficiência humana (HIV), agente causador da síndrome da imunodeficiência adquirida (AIDS ou SIDA) ${ }^{5}$.

A geração dos "baby boomers", pessoas nascidas entre 1945 e 1965, que agora entram na chamada $3^{\mathrm{a}}$ idade, traz novos comportamentos sobre o que significa "ser velho", particularmente em relação à sexualidade. Por exemplo, divórcio e taxas de re-matrimônio estão aumentando continuamente em idades tardias ${ }^{6,7}$; há uma tendência crescente de vida intima, mas de não-coabitação nas relações entre pessoas mais velhas; e existe uma maior aceitação legal e social de estilos de vida não-heterossexuais - lésbicas e gays mais velhos estão mais abertos em relação à sua identidade sexual ${ }^{6}$. Uma nova indústria está sendo implantada ao redor deste grupo de idosos, oferecendo serviços de encontros entre pessoas mais velhas e de aconselhamento sobre como formar relacionamentos íntimos na idade mais avançada ${ }^{8}$. Essas mudanças de valores, de atitudes e de comportamentos ficam ainda mais evidentes ao se constatar que as taxas de infecções sexualmente transmissíveis, inclusive o HIV, estão aumentando rapidamente entre pessoas com mais de 50 anos $^{6}$. No Brasil, com relação à faixa etária de 60 anos e mais, em 1998 a taxa de incidência era 2,8 e em 2010 a taxa é de $5,1^{9}$.

Portanto, em função da tendência registrada na literatura, o presente trabalho tem como objetivo conhecer as características gerais da AIDS em maiores do 60 anos e mais no Distrito Federal (DF).

\section{Material e Métodos}

Trata-se de um estudo retrospectivo, de caráter descritivo de série temporal onde foi analisada a tendência da AIDS em idosos no período de 1999 a 2009, o período de dez anos foi escolhido por apresentar um processo lento, mas consistente, de envelhecimento da população brasileira ${ }^{10}$.

Foi realizado no Distrito Federal, que contava em 2010 com uma população residente de 2.606.885 habitantes. Em 2010, a população residente com 60 anos ou mais chegou a 197.613 habitantes, sendo que $42,98 \%$ eram homens e $57,01 \%$ mulheres (dados da população residente, coletado do banco de dados do IBGE ${ }^{9,11}$ ).

Os idosos foram classificados por faixa etária em intervalos de 10 anos, agrupando, assim, as idades entre 60-69 anos, 70-79 anos e 80 anos e mais. Estas três faixas de idade são caracterizadas como idosos jovens, meio-idosos e idosos muito idosos, respectivamente $^{12}$, e são as faixas de idade disponíveis no Sistema de Informações de Agravos de Notificação (SINAN). O SINAN é um sistema de informação do Ministério da Saúde implantado em todo território nacional para monitoramento dos agravos de notificação compulsória.

A população do estudo foi constituída por todos os casos de AIDS notificados no SINAN relativos ao período de 1999 a 2009 ocorridos em pessoas com idade igual ou maior que 60 anos residentes no DF. Para evitar erros de retardo de notificação, optou-se por analisar os dados disponíveis até 2010, ano em que constavam os dados definitivos para o Distrito Federal. Estes dados foram obtidos do Sistema de Informações de Agravos de Notificação (SINAN) na Secretaria de Estado de Saúde do Distrito Federal (SES-DF).

$\mathrm{O}$ instrumento que alimenta o banco de dados são as fichas de notificações e investigação de AIDS estabelecida pelo o Ministério da Saúde e adotada em todo o Sistema Único de Saúde brasileiro. As fichas são preenchidas por profissionais de saúde e depois digitadas no SINAN.

O processamento e mapeamento dos dados foram realizados mediante utilização do programa TabWin (Tab para Windows), versão 3.6, software gratuito desenvolvido pelo DATASUS, que permite tabular diferentes tipos de informação em um mesmo ambiente, facilitando a construção de indicadores.

As variáveis abordadas neste estudo foram as seguintes: sexo, idade, categoria de exposição e ano do diagnóstico.

Para análise dos dados, foram elaborados relatórios com distribuições de frequências 
dos casos de AIDS. A razão entre o número de casos masculino/feminino foi calculada com o objetivo de avaliar mudança nesta relação ao longo dos anos. O cálculo das frequências absoluta e relativa, assim como o cálculo de incidência foram realizados utilizando-se o programa Excel, versão 7.0.

No calculo da incidência, utilizou-se a população de referência do senso do ano de 2010, ajustada pelo método direto ${ }^{1}$. O ajuste por idade, pelo método direto, é feito usando-se uma população padrão única, que funciona como um grupo comum de pesos para o cálculo de taxas ponderadas (ajustadas ou padronizadas). A população padrão utilizada foi a Distrito Federal $2010^{1,13}$. A taxa ajustada por idade foi calculada através da seguinte fórmula:

Taxa específica $=\frac{\sum\left(\begin{array}{c}\text { taxa específica } \\ \text { por idade }\end{array}\right) \times\left(\begin{array}{c}\text { população padrão do } \\ \text { DF na faixa etária }\end{array}\right)}{\sum_{\text {padrão DF }}^{\text {populão }}}$

O coeficiente de incidência serve para medir como uma doença, no caso a AIDS, está se comportando em uma população específica-aqui, todos os indivíduos maiores de 60 anos no Distrito Federal. A partir dele, pode-se analisar a importância que a AIDS revela nessa população. É válido lembrar que a taxa de incidência difere do número absoluto de casos, pois é sempre calculada de acordo com a população específica a ser estudada; consequentemente é através dela que se tem uma noção fidedigna de como a AIDS está se comportando na população acima de 60 anos.

Optou-se por calcular a incidência sobre a população geral menor que 60 anos e também na população especifica de maiores de 60 anos, para uma melhor análise do problema.

Aspecto ético: Por se tratar de um banco de domínio público, não foi necessário submeter o projeto ao Comitê de Ética em Pesquisa.

\section{Resultados}

Os dados referentes ao total de número de casos de AIDS diagnosticados no período de 1999 a 2009 totalizaram 4.258 casos novos, sendo que, destes, 89 ocorreram com idosos, representando 2,0\% do número total de casos. Embora seja uma porcentagem pequena, o crescimento anual foi contínuo, caindo nos anos de 2008 e 2009 (Tabela 1). ]

Tabela 1 - Distribuição dos casos de AIDS segundo ano de diagnóstico e faixa etária. Distrito Federal, 1999-2009.

Table 1 - Distribution of AIDS cases in the second year of diagnosis and age group. Federal District, 1999-2009.

\begin{tabular}{|c|c|c|c|c|c|}
\hline \multirow{2}{*}{$\begin{array}{l}\text { Ano } \\
\text { Diagnóstico }\end{array}$} & \multicolumn{2}{|c|}{$<60$ anos de idade } & \multicolumn{2}{|c|}{$>60$ anos de idade } & \multirow{2}{*}{$\frac{\text { Total }}{\mathrm{n}}$} \\
\hline & $\mathrm{n}$ & $\%$ & $\mathrm{n}$ & $\%$ & \\
\hline 1999 & 344 & 99,7 & 1 & 0,3 & 345 \\
\hline 2000 & 392 & 97,8 & 9 & 2,2 & 401 \\
\hline 2001 & 323 & 98,2 & 5 & 1,5 & 329 \\
\hline 2002 & 396 & 98,3 & 6 & 1,5 & 403 \\
\hline 2003 & 548 & 99,1 & 5 & 0,9 & 553 \\
\hline 2004 & 421 & 97,2 & 12 & 2,8 & 433 \\
\hline 2005 & 412 & 97,2 & 12 & 2,8 & 424 \\
\hline 2006 & 375 & 97,4 & 10 & 2,6 & 385 \\
\hline 2007 & 359 & 97,0 & 11 & 3,0 & 370 \\
\hline 2008 & 329 & 97,3 & 9 & 2,7 & 338 \\
\hline 2009 & 359 & 97,6 & 9 & 2,4 & 368 \\
\hline TOTAL & 4258 & 97,9 & 89 & 2,0 & 5173 \\
\hline
\end{tabular}

Fonte/Source: SINAN-GDST-AIDS/DIVEP/SVS/SES.

*Dados provisórios e parciais digitados até 02/01/2012 e obtidos das fichas de notificação/investigação de casos.

* Temporary and partial data input until January $2^{\text {nd }}, 2012$, and obtained from notification/case investigation files. 
Analisando-se as taxas de incidência no período de 1999 a 2009, dentro da população dos maiores de 60 anos residentes no DF, percebe-se que a taxa vem oscilando a partir de 1999, mesmo com o aumento dessa população, (Tabela 2) apresentando suas maiores incidências nos anos de 2000 e 2005 para ambos os sexos. Por se tratar de números pequenos, o coeficiente sofre um grande impacto, mesmo com um aumento pequeno no número absoluto e pode sofre variação aleatória nos anos por isto não podemos observar se realmente existe uma tendência de crescimento da epidemia no DF.

Com relação aos casos de AIDS segundo razão de sexo (M:F) e ano de diagnóstico, destaca-se o ano de 2006, quando ocorreu uma inversão da razão $(0,7: 1)$, resultando em mais casos nas mulheres do que nos homens nessa faixa etária. Esse indicador aponta para o crescimento do acometimento das mulheres idosas, mas é um dado que também tem flutuado muito, provavelmente influenciado pelos pequenos números.

Quando comparamos com a razão entre os indivíduos com menos de 60 anos de idade, observamos que ela vem se mantendo constante, o que contrasta com o mesmo indicador entre os idosos (Tabela 3 ).

Observa-se que, em relação à categoria de exposição e ao ano de diagnóstico por sexo, o maior número de casos nos homens, 27 (42,9\%), e nas mulheres, 21 (80,8\%), deu-se em decorrência de relação sexual heterossexual. Destaca-se que, em 22 (34,9\%) das notificações nos homens, os dados relativos ao tipo de exposição estavam incompletos e foram considerados ignorados. Sendo que nas mulheres, apenas $5(19,2 \%)$ estavam incompletos (Tabela 4).

Quanto à faixa etária, o grupo mais acometido foi aquele com idades entre 60 e 69 anos de idade. Em 1999, dentre os indivíduos nessa faixa etária o coeficiente de incidência foi de 1,7 casos por 100.000 hab.; já no ano seguinte, essa incidência chegou a 10,1 casos por 100.000 hab., e vem oscilando em torno desse patamar ao longo da série histórica analisada (Figura 1).

\section{Discussão}

Analisando-se a magnitude da AIDS, percebeu-se que a doença nessa população específica apresenta grande relevância

Tabela 2 - Casos de AIDS em maiores de 60 anos de idade (número e coeficiente por 100.000 hab.) segundo sexo e ano de diagnóstico. Distrito Federal, 1999 a 2009.

Table 2 - Cases of AIDS in over 60 years of age (by number and coefficient 100.000hab.) by sex and year of diagnosis. Federal District from 1999 to 2009.

\begin{tabular}{|c|c|c|c|c|c|c|}
\hline \multirow{2}{*}{$\begin{array}{l}\text { Ano de } \\
\text { diagnóstico }\end{array}$} & \multicolumn{2}{|c|}{ Masculino } & \multicolumn{2}{|c|}{ Feminino } & \multicolumn{2}{|c|}{ Total } \\
\hline & $\mathrm{n}$ & Coef. & & Coef. & $\mathrm{n}$ & Coef. \\
\hline 1999 & 1 & 2,5 & 0 & 0 & 1 & 1,11 \\
\hline 2000 & 8 & 16,4 & 1 & 1,6 & 9 & 8,21 \\
\hline 2001 & 3 & 6 & 2 & 3,2 & 5 & 4,46 \\
\hline 2002 & 6 & 11,8 & 0 & 0 & 6 & 5,23 \\
\hline 2003 & 4 & 7,7 & 1 & 1,5 & 5 & 4,27 \\
\hline 2004 & 7 & 13,2 & 5 & 7,5 & 12 & 10,05 \\
\hline 2005 & 11 & 19,9 & 1 & 1,4 & 12 & 9,62 \\
\hline 2006 & 4 & 7,1 & 6 & 8,5 & 10 & 7,85 \\
\hline 2007 & 6 & 8,7 & 5 & 5,3 & 11 & 6,77 \\
\hline 2008 & 8 & 10,8 & 1 & 1 & 9 & 5,09 \\
\hline 2009 & 5 & 6,4 & 4 & 3,7 & 9 & 4,8 \\
\hline Total & 63 & $\ldots$ & 26 & $\ldots$ & 89 & $\ldots$ \\
\hline
\end{tabular}


Tabela 3 - Casos de AIDS segundo razão de sexo (M:F) e faixa etária e ano de diagnóstico. Distrito Federal, 1999-2009.

Table 3 - Cases of AIDS second sex ratio (M: F) and age and year of diagnosis. Federal District, 19992009.

\begin{tabular}{lcc}
\hline \multirow{2}{*}{ Ano Diagnóstico } & \multicolumn{2}{c}{ Razão de sexo } \\
\cline { 2 - 3 } & $<60$ anos de idade & $>60$ anos de idade \\
\hline 1999 & $2,2: 1$ & $1,0: 1$ \\
2000 & $2,0: 1$ & $8,0: 1$ \\
2001 & $2,0: 1$ & $2,0: 1$ \\
2003 & $2,0: 1$ & $7,0: 1$ \\
2004 & $2,0: 1$ & $4,0: 1$ \\
2005 & $2,1: 1$ & $1,4: 1$ \\
2006 & $2,2: 1$ & $11,0: 1$ \\
2007 & $1,8: 1$ & $0,7: 1$ \\
2008 & $2,5: 1$ & $1,2: 1$ \\
2009 & $2,6: 1$ & $8,0: 1$ \\
\hline
\end{tabular}

Fonte/Source: SINAN-GDST-AIDS/DIVEP/SVS/SES.

*Dados provisórios e parciais digitados até 02/01/2012 e obtidos das fichas de notificação/investigação de casos.

* Temporary and partial data input until January 2nd, 2012, and obtained from notification/case investigation files.

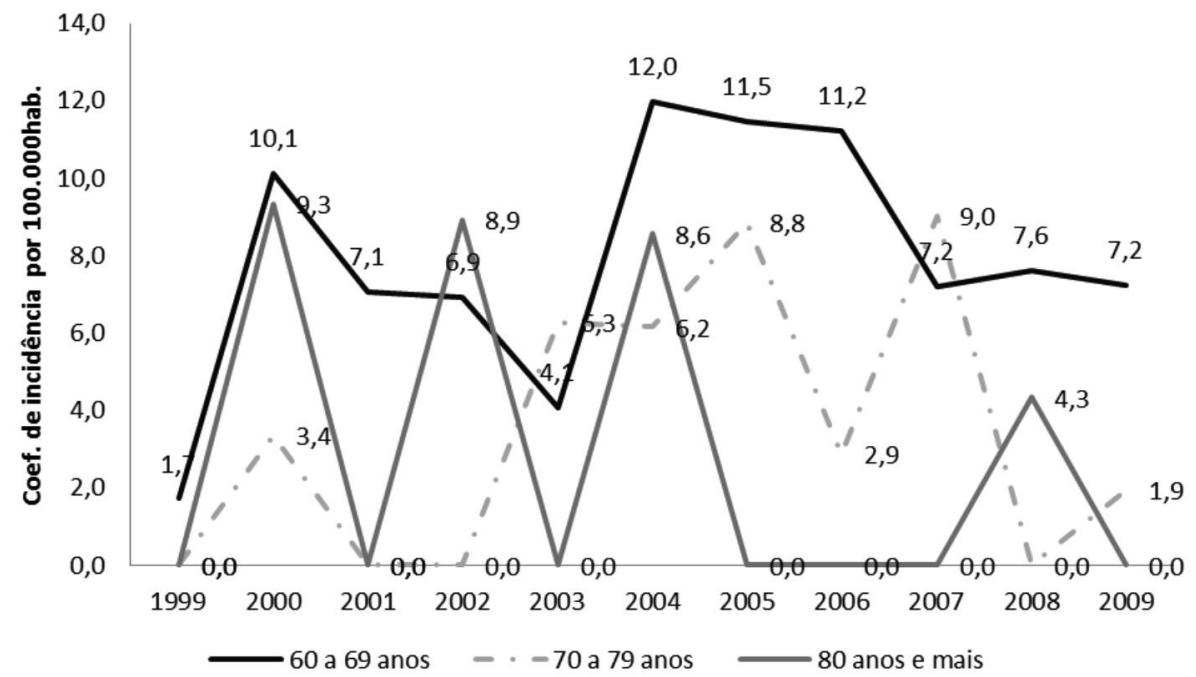

Figura 1 - Casos de AIDS em maiores de 60 anos de idade (número) segundo faixa etária e ano de diagnóstico. Distrito Federal, 1999 a 2009.

Figure 1 - Cases of AIDS in over 60 years of age (number) by age group and year of diagnosis. Federal District from 1999 to 2009.

epidemiológica, não pelos números absolutos, mas pelas taxas de incidência ano a ano.

Os dados coletados no Distrito Federal durante o período de 1999 a 2009 se diferenciam de forma global dos dados do Brasil, levando-se em consideração diferenças proporcionais ${ }^{10}$. Os resultados demonstram que, entre os velhos, o perfil da população mais afetado pela epidemia é o de homens de idade entre 60 a 69 anos, portanto idosos jovens. Podemos inferir que pelo menos uma parte da população estudada deve ter se infectado na faixa-etária de 50 a 60 anos, uma vez que da transmissão à situação de soropositivo, até a pessoa ser considerada um caso de AIDS podem decorrer de 5 a 10 anos.

Em maiores de 60 anos residentes no Distrito Federal pode-se perceber que, mesmo com o aumento dessa população, a taxa 


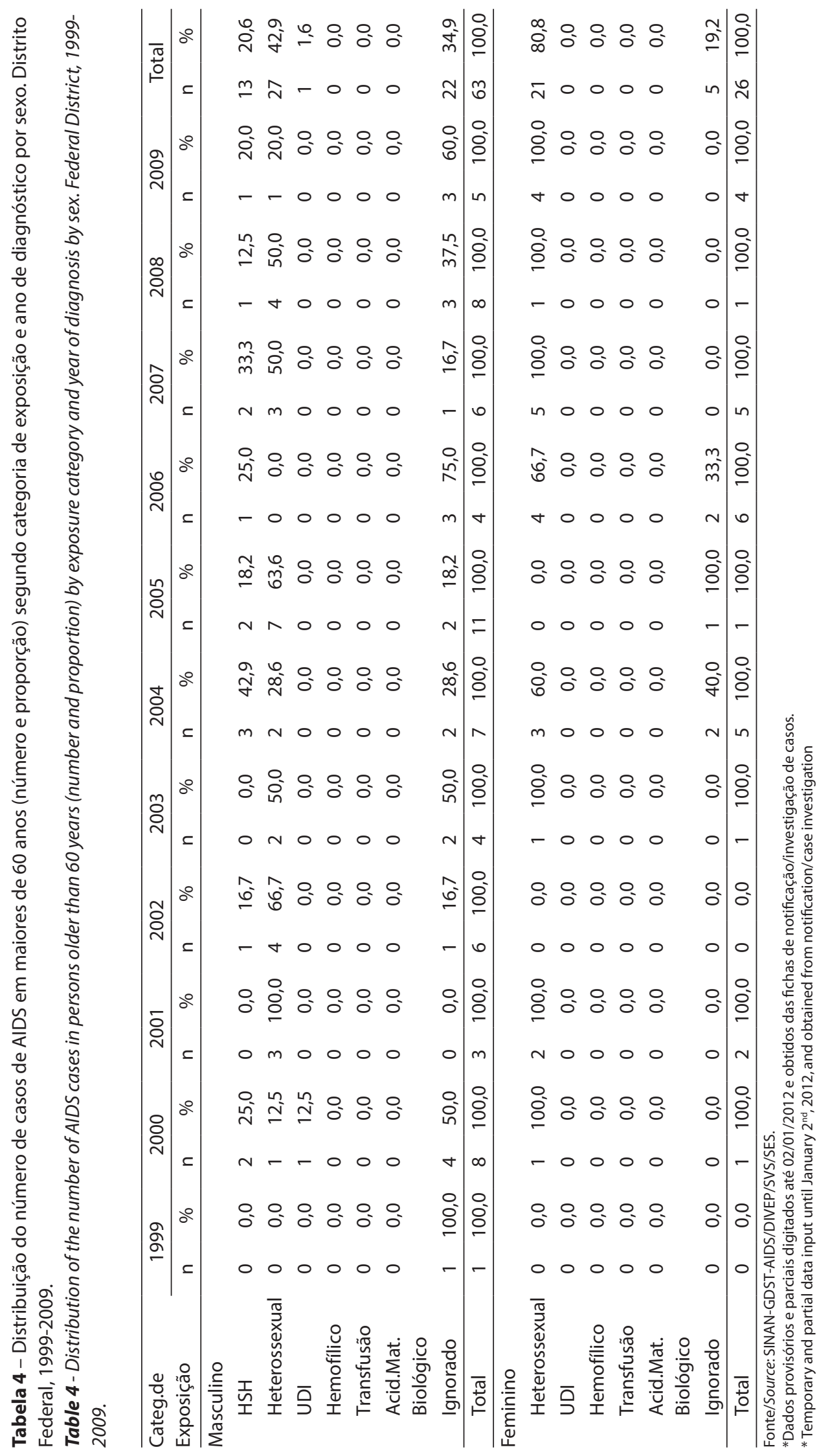


de incidência vem oscilando e apresentando suas maiores incidências nos anos de 2000 e 2005 para ambos os sexos.

Estes dados demonstram que, no Distrito Federal, os casos de AIDS não estão em consonância com o que acontece em todo Brasil, onde ocorreu um aumento significativo de casos entre 2000 e 2009. Segundo o boletim do Ministério da Saúde foram registrados 744 casos em 2000 e 1.623 em $2009^{14}$. Porém, se considerarmos a soma dos anos de 1999-2003, 26 casos, e de 2004- 2008, 54 casos, isso pode apontar um aumento nesta faixa-etária, não evidenciado ano a ano por se tratar de uma população menor.

A AIDS em idosos, no Distrito Federal, embora tenha um menor coeficiente de incidência de casos, quando comparada com outras faixas etárias, confere uma nova realidade à epidemia, especialmente quando observamos separadamente a faixa de 60 a 69 anos de idade (Figura 1).

Observa-se que algumas das características da epidemia, na população em geral, também são percebidas entre os idosos, dentre elas a heterossexualização relacionada, sobretudo, à transmissão sexual ${ }^{11,12,15}$. Dentre os fatores que podem estar contribuindo para esse novo perfil da epidemia destacam-se o aumento da atividade sexual entre os idosos, a disposição de tecnologia que melhora e prolonga a performance sexual, e a resistência em usar o preservativo $^{16,17}$. Quanto à feminização da epidemia, muitas mulheres idosas se sentem constrangidas em ter de pedir ao companheiro que use preservativo, pois isso prejudica a espontaneidade e acaba pondo em risco a relação sexual ${ }^{18}$.

Ainda em relação à categoria de exposição, mesmo que em pequeno número, chama a atenção o uso de drogas injetáveis entre os adultos mais velhos, grupo este que raramente é considerado tóxico-dependente. Os profissionais de saúde tendem a não investigar o uso de drogas nesta população, assumindo erroneamente que este grupo não é passível de tal comportamento ou, se o foi no passado, não se constitui fato relevante para o presente, no momento da consulta $^{15}$.

\section{Considerações Finais}

Conclui-se que a epidemia de HIV/AIDS pode ser considerada estável entre os idosos no Distrito Federal. Esse cenário pode estar mascarado pela dificuldade de diagnóstico oportuno de HIV para essa população.

Os vários fatos identificados por este estudo devem ser aprofundados, incentivando pesquisas comportamentais de vulnerabilidade relacionadas à infecção pelo HIV em idosos. Desse modo, será possível o monitoramento do comportamento sexual de risco, com vistas à intervenção na epidemia, e, consequentemente, a redução da morbimortalidade por AIDS na terceira idade.

Embora a avaliação da qualidade dos dados segundo características do indivíduo não tenha sido objeto de análise, observou-se que, na distribuição proporcional segundo sexo, a falta de informação sobre a forma de transmissão do vírus HIV foi superior entre indivíduos adultos do sexo masculino, indicando dificuldade na obtenção de dados sobre os antecedentes epidemiológicos do paciente.

É provável que a falta de informação esteja relacionada à maior dificuldade de classificação em categorias estigmatizadas, como homens que fazem sexo com outros homens e usuários de drogas injetáveis.

Atualmente, a notificação compulsória é a principal ferramenta de vigilância epidemiológica do HIV/AIDS no Distrito Federal e no Brasil. Entretanto, é necessário salientar que essas informações estão sujeitas a erros decorrentes de digitação e de registro, além de possíveis subnotificações na base de dados no Sistema de Informações de Agravos de Notificação - SINAN. Tais limitações são inerentes aos dados de origem secundária.

Em relação à subnotificação, destaca-se que os antirretrovirais são medicamentos de alto custo, estão disponíveis gratuitamente no Sistema Único de Saúde - SUS e não são comercializados pela iniciativa privada no 
Brasil. Esse aspecto, em conjunto com a portaria distrital que estabelece a notificação como um dos documentos a serem apresentados pelo usuário no momento do cadastro para dispensação dos medicamentos antirretrovirais, colabora para a detecção mais fidedigna dos casos de AIDS no DF ${ }^{27}$.

No DF anualmente são desenvolvidas campanhas de conscientização sobre transmissão de DST direcionadas à população em estudo. Um exemplo desta ação é a publicação de anúncio, apresentado diariamente (períodos de campanhas de DST) nos classificados (seção de acompanhantes) de todos os jornais do DF sobre cuidados com a prevenção da transmissão do HIV.

Sugere-se que haja uma continuidade destas ações, bem como se implementem novas estratégias de prevenção que possibilitem maior conhecimento da população acima de 60 anos acerca da sua vulnerabilidade e que devem ser incorporadas à rotina dos serviços.

\section{Referências}

1. Instituto Brasileiro de Geografia e Estatística. Ibge. Síntese de Indicadores Sociais. Uma análise das condições de vida da população brasileira - 2010. Rio de Janeiro: IBGE; 2010. Disponível em: http:/ / www.ibge. gov.br/home/estatistica/populacao/condicaodevida/ indicadoresminimos/sinteseindicsociais2010/default. shtm. [Acessado em 21 de maio de 2012]

2. Organização Mundial da Saúde; Envelhecimento ativo: uma política de saúde. Brasília. 2005. Disponível em: http:/ / www.who.int/es/ [Acessado em 20 de maio de 2012]

3. Groisman DA. Velhice entre o normal e o patológico. História, Ciências e Saúde - Manguinhos jan-abr 2002; 9: 1 e 61-78. Disponível em: http:/ /www.scielo.br/pdf/ hcsm/ v9n1/a04v9n1.pdf. [Acessado em 21 de maio de 2012]

4. Gott M. Sexual health and the new ageing. Age Ageing 2006; 35(2): 106-7.

5. Aquino EML. Saúde do homem: uma nova etapa da medicalização da sexualidade? Ciênc Saúde Colet 2005; 10(1): 19-22.

6. Potts A, Gavey N, Grace, V, Vares T. The downside of Viagra: women's experiences and concerns about Viagra use by men. Sociology of Health \& Illness 2003; 25(7): 697-719.

7. Zornitta M. Os novos idosos com AIDS e desigualdade à luz da bioética [dissertação de mestrado]. Rio de Janeiro: Escola Nacional de Saúde Pública Sergio Arouca; 2008.

8. Munk RJ, Jenison SA. Older people and HIV. New México: AIDS Education and Trainig Center. Disponível em: www.AIDSinfonet.org. [Acessado em 21 de maio de 2012]

9. Brasil. Ministério da Saúde. Secretária de Vigilância Saúde. Programa Nacional de DST e AIDS. Boletim Epidemiológico - AIDS e DST - Versão Final - Ano VII. no 1 - 27 a $52^{\text {a }}$ semanas epidemiológicas - julho a dezembro de 2009 e $01^{\text {a }}$ a $26^{\text {a }}$ semanas epidemiológicas - janeiro a junho de 2010. Disponível em: http:// www.AIDS.gov.br/sites/default/files/ anexos/ publicacao/2010/45974/vers_o_final_15923.pdf. [Acessado em 21 de maio de 2012]

10. Emlet, C A. Lessons Learned. Moving forward. Tacoma: University of Washington; 2005.

11. IBGE. Censo demográfico. Disponível em: http://www. ibge.gov.br. [Acessado em 2 de janeiro de 2012]

12. Veras RP. País jovem com cabelos brancos: a saúde do idoso no Brasil. Rio de Janeiro: Relume Dumará; 1994.

13. Szklo M, Nieto FJ. Epidemiology beyond the basics. Gaithersburg: Aspen Publishers; 2000.

14. Ribeiro AM, Oliveira MLC, Melo GF. A AIDS de cabelos brancos: ocorrência de AIDS em idosos no Brasil. (no prelo)

15. Santos NJS, Tayra A, Silva SR, Buchalla CM, Laurenti R. A AIDS no Estado de São Paulo: As mudanças no perfil da epidemia e perspectivas da vigilância epidemiológica. Rev Bras Epidemiol 2002; 5(3): 286-310.

16. Melo MR, Gorzoni M, Melo KC, Melo E. Síndrome da imunodeficiência adquirida no idoso. Rev Diag Trat 2002; $7:$ 13-7.

17. Savasta AM. HIV: Associated Transmission Risks in Older Adults - An Integrative Review of the Literature. J Assoc Nurses Aids Care 2004; 12 (1): 50-9.

18. Brasileiro M, Freitas MIF. Representações sociais sobre AIDS de pessoas acima de 50 anos de idade, infectadas pelo HIV. Rev Latino-Am Enfermagem 2006; 14(5): 78995.

19. UNAIDS. AIDS epidemic update. 2012. Disponível em: http://www.unAIDS.org. [Acessado em 1 de julho de 2009]. 
20. Distrito Federal. Secretaria de Estado de Saúde. Subsecretaria de Vigilância em Saúde. Diretoria de Vigilância Epidemiológica. Gerência de DST/AIDS e HV. Boletim Epidemiológico de DST/AIDS do Distrito Federal. Brasília, DF. Número 1/2011.

21. IBGE. Censo demográfico. Disponível em: http://www. ibge.gov.br. [Acessado em 2 de janeiro de 2012]

22. Vasconcelos EMR, Alves FAP, Moura LML. Perfil epidemiológico dos clientes HIV/AIDS na terceira idade. Rev Bras Enferm 2001; 54(3): 435-45.

23. Toledo LSG, Maciel ELN, Rodrigues LCM, Tristão-Sá R, Fregona G. Características e tendência da AIDS entre idosos no Estado do Espírito Santo. Rev Soc Bras Med Trop 2010; 43(3): 264-7.

24. Links NL. HIV older adults: age-specific issues in prevention and treatment. AIDS Read 2000; 10(7): 43040.
25. Godoy VS, Ferreira MD, Silva EC, Gir E, Canini SRMS. O perfil epidemiológico da AIDS em idosos utilizando sistemas de informações em saúde do DATASUS: realidades e desafios. DST - J Bras Doenças Sex Transm 2008; 20(1): 7-11.

26. De Lorenzi DRS, Saciloto B. Frequência da atividade sexual em mulheres menopausadas. Rev Assoc Med Bras 2006; 52(4): 256-60.

27. Portaria nº. 37, de 23 de março de 2010. Estabelece os documentos que deverão ser fornecidos pelo médico assistente ao paciente para fins de cadastramento nas unidades de saúde dispensadoras de medicamentos antiretrovirais. Diário Oficial do Distrito Federal, 25 de março de 2010, No 58, p.10.

Recebido em: 05/01/12

Versão final apresentada em: 18/07/12

Aprovado em: 29/11/12 